\title{
Planejamento do AVEA Adequado à Realidade Institucional: o Caso do Núcleo de Educação à Distância (NED) do Senai/Sc
}

Aline Pereira Soares - UFSC - alinepsoares@ yahoo.com.br Marina Keiko Nakayama - UFSC - marina@egc.ufsc.br

Fernando José Spanhol - UFSC - spanhol@led.ufsc.br

Andressa Sasaki Vasques Pacheco - UFSC - andressa@ead.ufsc.br

Patrícia Sá Freire UFSC - aline@ led.ufsc.br

Maurício Rissi - UFSC - mauriciorissi@ead.ufsc.br

Gustavo Lucas - CESUSC - $\underline{\text { lucas.gustavo87@gmail.com }}$

\section{RESUMO}

Este trabalho objetivou analisar e identificar se o Ambiente Virtual de Aprendizagem AVEA - utilizado está adequado à realidade da equipe (e dos alunos) do Núcleo de Educação à Distância (NED) do Senai/SC-Florianópolis ao avaliar a opinião dos Coordenadores, Tutores e Monitores. Quanto aos procedimentos metodológicos caracteriza-se como teórico-empírico, descritivo, ex-post-facto, aplicado, bibliográfica e estudo de caso, com abordagem predominantemente qualitativa. Conclui-se, portanto, que o AVEA necessita de mais ferramentas interativas síncronas, melhores formas de armazenar as informações (para que a equipe consiga pelo ambiente, trocar informações mais estratégicas) e sugere-se a utilização de uma ferramenta que possibilite maior participação da Equipe na escolha ou melhoria do AVEA.

Palavras-chave: AVEA. Educação à Distância. Avaliação

\section{ABSTRACT \\ PLANNING OF THE ADEQUATE AVEA TO THE INSTITUCIONAL REALITY: THE CASE OF THE NUCLEUS OF DISTANCE EDUCATION (NED) OF THE SENAI/SC}

This work objectified to analyze and to identify if Virtual Environment of Learning the used is adjusted to the reality of the team (and the pupils) of the Nucleus of Distance Education (NED) of the Senai/SC-Florianópolis when evaluating the opinion of the Coordinators, Tutors and Monitors. The metodologics procedures it is characterized as: theoretician-empiricist characterizes itself as, description, ex-post-facto, applied, bibliographical and study of case, with predominantly qualitative boarding. One concludes, therefore, that the AVEA needs more synchronous interactive tools, better forms to store the information (so that the team obtains for the environment, to change more strategical information) and suggests it use of a tool that makes possible greater participation of the Team in the choice or improvement AVEA.

Key-words: AVEA. Distance Education. Evaluation.

\section{Introdução}

É uma realidade que para o processo de ensino-aprendizagem, a adoção do Ambiente Virtual de Aprendizagem (AVEA) promoveu um impacto significativo "abrindo-lhe perspectivas novas de acesso ao conhecimento universal e possibilitando uma interessante maneira de produzir conhecimentos" (SEE/ PROINESP/MEC). Mas, devido o aumento da oferta de cursos à distância, passa a ser uma necessidade crescente, o Pensar no Planejamento do uso do AVEA no processo de gestão das informações para o melhor desempenho das equipes de tutores e monitores. Vale ressaltar a oferta realizada em agosto do ano corrente pelo Governo Federal, de trinta e seis mil vagas 
para capacitação e formação continuada no uso das novas tecnologias da informação e da comunicação (AVEA e outras) no âmbito pedagógico (SEED/MEC).

Sendo assim, avaliar com diferentes perspectivas críticas o AVEA confirma-se cada vez mais como tema relevante. E criar estratégias que aperfeiçoem o sistema a partir da própria equipe é um desafio atual no processo de Educação à Distância - EAD.

Invariavelmente as Tecnologias de Informação e Comunicação (TIC's), inclui-se o AVEA, são um meio de transportar conhecimento em redes de interações gerenciadas por vezes sem foco analítico no indivíduo, mas nos relacionamentos, o que nos leva a repensar como esses ambientes podem ser socialmente construídos. Pode-se também dizer que eles são circulares, ou seja, funcionam em termos de circularidade produtiva. E nesse giro de "produção de riqueza", observa-se que há um centro propagador de informação muito mais relevante do que dados inseridos num banco de informações: o individuo. Nesse sentido têm-se teorias propostas por Donald Norman (1986) e seus trabalhos sobre a Tecnologia Centrada no Ser Humano, que propõe um novo modelo de pensar a tecnologia, considerando aspectos importantes da Psicologia Cognitiva e do Design de Interfaces. A Psicologia Cognitiva estuda os processos perceptivos e a forma como o ser humano percebe as coisas no contexto que está inserido, tentando explicar porque alguns estímulos são mais bem lembrados do que outros, a capacidade da memória, os tipos e modelos mentais básicos para explicar como os seres humanos interagem com os objetos/máquinas na Sociedade do Conhecimento.

Deste modo, a utilização do AVEA torna-se uma ferramenta estratégica não só para o aluno, mas especialmente para a Equipe de Coordenadores, Tutores e Monitores, sendo estes o meio de contato entre aluno e instituição, tendo posse de informações das necessidades dos discentes. E para gestão destas informações e de outras demandas advindas do processo de ensino à distância, as Instituições precisam adotar uma nova postura: a gestão que gere conhecimento para todos os agentes do processo, o que se leva a olhar a infra-estrutura tecnológica como um caminho facilitador deste trabalho. Faz-se então necessário a resolução de medidas que torne a informação e o conhecimento algo que possa ser compartilhado: planejamento, compartilhamento de informações, treinamento da equipe, gerenciamento das informações, são variáveis que viabilizam o processo de trabalho (Terra, 2002).

Precisa-se de estratégias alinhadas com o interesse da Instituição, ou seja, infraestrutura física e digital que favoreçam e estimulem o compartilhamento, a criação e o uso do conhecimento. Desse modo, uma vez que a equipe de trabalho manipula um grande volume de informações e interage com um alto número de alunos, torna-se fundamental o planejamento do AVEA visando proporcionar sua melhor utilização para o desenvolvimento e sucesso de todo o processo de EAD.

Dentro desta premissa percebeu-se a necessidade de avaliar se o AVEA adotado no NED/SENAI/SC possibilita que as áreas internas formem uma rede de relacionamentos, tanto locais (escrito e concreto) como virtuais (sensorial e dinâmico), desenvolvendo uma sinergia entre: corporações, inovações tecnológicas, estratégias, aprendizagem, conhecimento, pessoas e comunicação. E baseados no pressuposto da significativa relação entre - AVEA, avaliação do sistema com base no usuário e o bom desempenho de equipe e dos alunos - elaborou-se a questão da pesquisa: Como o planejamento do AVEA utilizado pela equipe do Núcleo de Educação à Distância (NED) do Senai/Sc-Florianópolis pode ser mais adequado à realidade Institucional?

Para tanto o objetivo geral da pesquisa é identificar se o AVEA utilizado está adequado à realidade da equipe e dos alunos do Núcleo de Educação à Distância (NED) do Senai/Sc-Florianópolis avaliando o modo pelo qual foi adotado. E específicos são: a) analisar as principais Tic's utilizadas no AVEA identificando e comparando pontos 
fortes, fracos, oportunidades e ameaças; b) analisar se as principais necessidades/ exigências dos componentes da equipe são viabilizadas pelo AVEA, ou seja, como é feito todo planejamento do uso do AVEA na equipe; c) avaliar o modo que o AVEA é utilizado - se elas propiciam a troca efetiva de informações e da gestão do conhecimento da equipe. Desta forma, foi eleito como caso de estudo a equipe de Coordenadores, Tutores e Monitores do Núcleo de Educação à Distância (NED) do Senai/ScFlorianópolis.

\section{Fundamentação Teórica}

\subsection{A Tecnologia - AVEA - no Processo de Trabalho da Equipe de EAD}

Segundo destacam Nakayama, Binotto e Pilla (2006, p.100) o surgimento dos microcomputadores que possibilitou a oferta de acesso à rede por um custo mais baixo, levou ao "despertar do interesse das pessoas pela internet". Em 1995 iniciou-se o acesso comercial à internet no Brasil e seus efeitos começaram a ser sentidos em todos os setores da sociedade. Estas novas formas de trabalhos virtuais são as que "permitem a produção e/ou execução de tarefas e serviços à distância sem que os trabalhadores estejam no ambiente físico da empresa" (Nakayama, Binotto e Pilla, 2006, p.100).

Para a educação, este recurso veio ampliar não somente o mercado de trabalho à distância (fora do local físico da empresa), mas principalmente, atender às necessidades da Sociedade do Conhecimento e a imensa demanda de profissionais em estudar a qualquer tempo e em qualquer lugar, permitindo-os construir conhecimentos básicos demandados pelo mercado de trabalho, recuperar conhecimentos perdidos e, promover sua educação continuada, exigências do mundo globalizado e altamente competitivo. Como afirma Spodick (2008), a EAD deve ser vista como um sistema provedor das oportunidades educacionais necessárias para todos os interessados, em qualquer lugar e a qualquer tempo, e com recursos necessários para que se complete competentemente a construção do conhecimento. Recursos que seriam: contato direto entre o professor e seus alunos; aprendizagem ativa por meio de respostas do aluno; realimentação ágil do grau de compreensão e desempenho do aluno; oportunidade de o aluno fazer revisões e aprender com seus próprios erros.

Neste cenário, cresce a importância dos recursos que as Tic's têm para o processo de $\mathrm{EAD}$, principalmente por meio das ferramentas pedagógicas que proporcionam aos alunos e educadores o aprimoramento em meio ao contato com as inovações, diferentemente das outras linguagens de aprendizagem as quais estão familiarizados. Não aperfeiçoar os recursos é se afastar do status de Sociedade do Conhecimento, e inclusive afastar os educadores e alunos brasileiros da participação e atualização necessárias para o seu crescimento competitivo.

O grande volume de informações pelos quais os diferentes tipos de equipes de EAD têm acesso deve-se às bruscas mudanças pelas quais passaram e passam depois da chamada Revolução da Informação, em especial com o desenvolvimento das Tic's. De acordo com Castells (1999), fomos impactados pela Revolução da Informação que exige novas dinâmicas, e conseqüentemente, impacta o como as organizações trabalham a informação. Segundo o autor, os principais aspectos dessa nova revolução são quatro: (1) "a informação é a matéria-prima" por serem as tecnologias agentes sobre a informação, e não apenas informação para agir sobre a tecnologia, como nas revoluções anteriores; (2) "penetrabilidade dos efeitos das novas tecnologias", pois como a informação é uma parte integral da atividade humana, os processos individuais e coletivos são diretamente moldados, embora não determinados, pelos novos meios tecnológicos; (3) "lógica de redes", onde em qualquer sistema ou conjunto de relações 
abre-se facilmente a possibilidade de acesso ao outro com as novas TI's, pois morfologia da rede parece adaptar-se à crescente complexidade de interação e aos modelos imprevisíveis do desenvolvimento derivado do poder criativo dessa interação. (4) "paradigma da TI é baseado na flexibilidade", visto que não apenas os processos são reversíveis, mas organizações e instituições podem ser modificadas, e até mesmo fundamentalmente alteradas pela reorganização de seus componentes.

Neste cenário, a troca e gestão de informações pelo AVEA tornam-se fator fundamental no processo de educação. A sua importância deve-se inclusive pelo investimento em Tic's, ou AVEA, ser base para um País, com as diferentes Instituições e suas equipes, ser considerado intensivo em conhecimento. Segundo Evers (2001), para que tal processo se concretize, a sociedade precisa investir em variáveis que separam os países com ou sem status de Sociedade do Conhecimento, como: investimento em pesquisa e desenvolvimento, computadores por pessoa, acesso á internet, número de cientistas e engenheiros (P\&D), investimento/desenvolvimento de infra-estrutura tecnológica (TIC), dentre outros.

No Brasil as Instituições têm vários tipos de softwares para AVEA disponíveis para utilização livre (licenciamento livre) ou comercial (licenciamento proprietário). Entretanto, para um efetivo desenvolvimento do AVEA, faz-se necessário acompanhar a tendência e o interesse de desenvolver tecnologias com foco cada vez maior no usuário (no consumidor, no homem), objetivando identificar o atendimento às suas reais necessidades. Portanto, o software utilizado pelo SENAI para o desenvolvimento do AVEA precisa inclusive pensar nos usuários.

\subsection{O uso do AVEA nas Instituições de Ensino}

Para Dougiamas (2008) o criador de um dos ambientes de aprendizagem mais conhecidos, o Moodle, pela perspectiva do usuário (aluno de EAD) as principais características são: a facilidade de navegação, o design fácil e atrativo da página e, as possibilidades de interatividade. Para o professor, as regras necessárias seriam as que possibilitem construir os próprios conteúdos, modificando-os se necessário, aprender mais sobre cada aluno e acompanhar o seu desenvolvimento individual e do grupo. $\mathrm{O}$ autor afirma inclusive que o grande desafio para o desenvolvimento e utilização do AVEA está em ajudar as pessoas (alunos e professores) no como utilizar a tecnologia e, desenvolver novas tecnologias como instrumentos facilitadores desta mesma aprendizagem.

Atualmente parece uma unanimidade afirmar que a efetividade das trocas das informações pelo AVEA pode contribuir para a competitividade organizacional - o que demonstra a importância das pessoas e equipes como possuidoras e criadoras de conhecimento e faz as organizações compreenderem que o conhecimento reside menos em máquinas e mais nas pessoas (Brown e Duiguid, 2001), não estando totalmente presente individualmente e nem na organização, mas compartilhado em cada um deles (ambos são complementares). Essa nova visão permite priorizar o conhecimento do usuário - seja ele aluno ou professor

Assim, os diversos tipos de mídia utilizados na Educação à distância dependem de múltiplos fatores: a facilidade de acesso dos alunos, a clareza para se passar os conteúdos, a naturalidade do professor para trabalhar com esses recursos, o custo por e para cada aluno e a facilidade de comunicação entre todos os envolvidos, especialmente no processo de aprendizado, já que educação tem como principal base a comunicação e a transmissão das informações para a construção do conhecimento (Oliveira, 2000). 
Para Santos e Rodrigues (1999) os serviços de comunicação podem ser classificados em dois tipos: síncronos: exigem que os interlocutores estejam conectados no mesmo momento temporal para que possa haver comunicação; e assíncronos: os momentos de envio e recepção de mensagens é em tempo diferente. As Mídias Assíncronas, segundo Silva (2008) possuem as seguintes características: flexibilidade acesso ao material didático em qualquer lugar e a qualquer hora; tempo para reflexão o tempo poderá ser potencializado para a reflexão sobre o material didático proposto, tempo para ter idéias e preparar os retornos, verificar as referências bibliográficas e possibilidade de acesso ao material quantas vezes for necessário e facilidade de estudo. Além de possibilitar a administração dos estudos de forma a aproveitar todas as oportunidades de tempo, seja no trabalho ou em casa, podendo ocorrer à integração de idéias e discussão sobre o curso em fóruns específicos. No caso das mídias síncronas, Silva (2008) relata que as características são: uma comunicação e respostas espontâneas; motivação - evidencia a sinergia dos trabalhos individuais e em grupo e encoraja os estudantes a criarem e continuarem seus estudos e presença - fortalece o sentimento de comunidade.

\section{Procedimentos Metodológicos}

Quanto ao tipo de estudo, caracteriza-se como descritiva, documental, bibliográfica, teórico-empírica, estudo de caso. Em relação à abordagem classifica-se como predominante qualitativa.

Nesta pesquisa foram coletadas informações do curso a distância em capacitação do corpo técnico do Serviço Nacional de Aprendizagem Industrial de Santa Catarina.

Quanto ao tipo de corte, esta pesquisa é transversal ou cross-sectional, neste caso, com os tutores participantes do segundo semestre de 2008. Os dados primários foram coletados junto a todos os tutores/monitores e coordenadores do curso, utilizando-se assim um censo. Foram aplicados 9 questionários composto de questões abertas e fechadas com escala de avaliação verbal. Cada questão correspondia a uma das variáveis em estudo, conforme o quadro 1, exposto a seguir:

\begin{tabular}{|c|c|}
\hline Questão & Variável \\
\hline 1 & TIC's adotadas - AVEA \\
\hline 2 & Tempo que é usuário \\
\hline 3 & Planejamento de adoção do AVEA \\
\hline 4 & Avaliação AVEA \\
\hline 5 & Pontos Fortes do AVEA \\
\hline 6 & Pontos Fracos do AVEA \\
\hline 7 & Sugestões de Melhorias do AVEA \\
\hline 8 & Estratégias de treinamento para utilização do AVEA \\
\hline 9 & Dificuldades encontradas no AVEA \\
\hline 10 a 13 & Tendêncosias de sistemas (AVEA) em EAD \\
\hline 14 & AVEA como facilitador do compartilhamento de informações \\
\hline 15 & Fonte: Dados primários \\
\hline \multicolumn{2}{|c|}{} \\
\end{tabular}

Após a coleta de dados há a seção de tratamento. As estratégias de análise adotada foram: qualitativa, descritiva e interpretativa com a exposição das médias. Optou-se pela utilização da unidade descritiva média para a comparação entre os termos. Destaca-se que esta média tem como valor mínimo zero e valor máximo quatro, sendo quanto maior o valor, maior a será o compartilhamento de informações da equipe proporcionadas pelo AVEA. Apesar de não ser cientificamente suficiente, é uma importante estratégia de análise já que auxilia o pesquisador a entender seu objeto de 
pesquisa. Devido a essa deficiência, complementou-se também com a análise de discurso.

\section{Curso de EAD do Senai}

Diante da demanda motivada pela competitividade, o Serviço Nacional de Aprendizagem Industrial de Santa Catarina (SENAI/SC) se preocupou em capacitar os funcionários de diversas indústrias brasileiras quanto para o enfrentamento da acelerada mudança de tecnologias, processos. Segundo o site do curso a distância, o "Centro de Tecnologia em Automação e Informática do SENAI/SC tem como objetivos a geração e difusão de tecnologia em Automação e Informática, visando auxiliar o avanço das empresas brasileiras em um mercado de alta competitividade mundial". O curso de capacitação por meio da $\mathrm{EaD}$, tem como objetivo atualizar o conhecimento técnico dos colaboradores do SENAI.

A carga horária total do curso é de 332 (trezentos e trinta e duas) horas/aulas, sendo a maior parte ministrada a distância e não mais que 40 horas presencial, que constituem-se de encontros, seminários temáticos e exames.

O curso conta com a seguinte equipe de apoio: 1 Coordenador de Monitores, 1 Coordenadora de Projetos, 11 Consultores no Núcleo de Gestão Empresarial do SENAI e 7 Tutores - Conteúdistas (1 para cada disciplina). Destaca-se a função dos monitores e tutores, facilitadores da aprendizagem, motivando os estudantes para as atividades, esclarecendo dúvidas, reforçando aprendizagem e coletando informações sobre os estudantes (desempenho, notas, atividades realizadas, opiniões e sugestões). São eles os responsáveis pelo envio, recebimento e correção de atividades ao seu grupo de estudantes. Vale ressaltar que cada monitor é responsável por um grupo de 120 alunos.

Moore e Kearsley (2007) afirmam que para a constituição da equipe de EAD faz-se necessário que o sistema possua: fonte de conhecimento que deve ser ensinada e aprendida; um subsistema para estruturar esse conhecimento em materiais e atividades para o aluno (denominado cursos); outro subsistema que transmita os cursos para os alunos; um subsistema que controle e avalie os resultados de modo que quando ocorrerem falhas, intervenções sejam possíveis e, uma organização com uma política e uma estrutura administrativa para ligar essas peças distintas.

\section{Descrição dos Resultados}

Ao analisar os resultados "relacionado ao tempo que é usuário", percebe-se que todos os componentes utilizam o sistema há pelo menos 1 ano (com média de 3 anos) o que demonstra um nível de usabilidade alto e os qualifica para opinarem sobre a questão da pesquisa.

A Escolha do AVEA: As TIC's adotadas, no caso o AVEA utilizado pelo EAD/SENAI, denomina-se SENAI Virtual. É um programa desenvolvido pela Datasul especialmente para a instituição. Ou seja, o processo de desenvolvimento do software foi especifico e pretendeu atender necessidades e demandas específicas do processo de EAD da organização.

O Planejamento: O "Planejamento de adoção do AVEA" pelo SENAI foi elaborado a partir da percepção da coordenação de que inicialmente quando usavam uma plataforma de ensino própria não estavam obtendo o bom desempenho pretendido devido às especificidades e exigências de cada curso cursos. A partir desta percepção, chegou-se a decisão de buscar um novo AVEA que atendesse não somente as necessidades básicas de um processo de ensino-aporendizado virtual, mas também que aperfeiçoasse sua 
utilização, possibilitando a satisfação das exigências de cada curso especificamente. Deste modo a coordenação solicitou um programa mais robusto que foi desenvolvido por uma empresa especializada - Datasul - que após vários encontros com a equipe de coordenadores visando levantar detalhes sobre as necessidades e resultados pretendidos para o ambiente, elaborou o software com página inicial apresentado na Figura 1. Vale apontar que, para não expor informações estratégicas, a página retratada na Figura 1 não contém todas as informações de um curso comum.

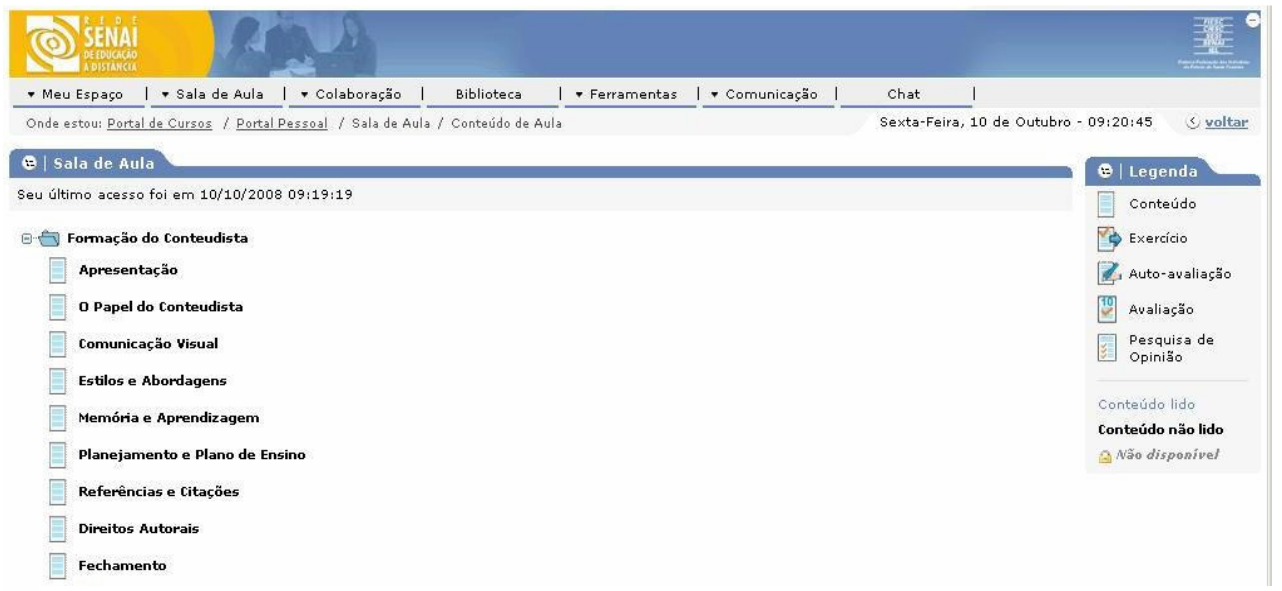

Figura 1 - Pagina Principal do AVEA - Senai Virtual

Fonte: (SENAI, 2008)

Os componentes da equipe avaliam como "boa" a escolha do AVEA. Esta constatação confirma-se devido aos nove (todos) participantes classificarem positivamente a avaliação dos softwares adotados pela Instituição. Afirmam também que a Coordenação da equipe se compromete em realizar avaliações do sistema periodicamente (pelo menos 1 vez ao mês). Considerando a teoria, constata-se: as equipes que fazem "mensuração dos resultados das estratégias" cumprem um dos quesitos para a troca efetiva de informações e de conhecimento. Entretanto, quando questionados quanto ao critério utilizado para o planejamento de adoção do AVEA, a maioria (6) afirma que o foco principal "foi o atendimento a necessidade do cliente", enquanto outros três consideraram que "a alta administração escolheu o sistema e solicitou apenas que eles a colocassem em uso". Segundo os entrevistados, o planejamento é realizado pela unidade gestora do SENAI/SC - departamento Regional (SENAI-DR) onde as unidades não participam desse planejamento, o que levanta a questão de que pode haver pontos a melhorar visto que, pelo menos alguns membros da equipe, que já utilizavam o antigo programa, poderiam responder uma avaliação para adoção do programa atual.

A escolha foi pela funcionalidade da plataforma de ensino, facilidade de navegação, preço (licitação). Inicialmente usávamos uma plataforma própria, mas devido o grande número de alunos advindos de diversos projetos de atendimento às corporações, foi necessário adquirir um software mais adequado às necessidades dos alunos.

As principais ferramentas planejadas para o compartilhamento das informações foram o e-mail mencionado por quatro (4) entrevistados, dois (2) a conversa com os colegas, dois (2) pelo Chat, e um (1) mencionou o fórum. O planejamento é realizado pela unidade gestora do SENAI - departamento Regional (SENAI-DR) onde as unidades não participam desse planejamento.

Treinamento para utilização do AVEA : Quanto às estratégias de treinamento para utilização do AVEA, sete (7) afirmam que receberam treinamento de 2 horas onde o 
sistema e suas ferramentas foram apresentados e dois (2) indicam que não receberam treinamento algum e que aprenderam com os colegas ao usarem o sistema. Ou seja, no geral percebe-se que não houve um planejamento efetivo com simulações para que o sistema fosse implantado.

Pontos Positivos do AVEA: Os pontos positivos do sistema concentram-se na interatividade, gerenciamento e na possibilidade de criar diferentes atividades para avaliar os alunos: quatro (4) elogiam a interatividade, outros quatro (4) no gerenciamento dos dados dos alunos (facilidade de exportar dados, compartilhar documentos, controle dos e-mails enviados, gerar relatórios) e também três (3) mencionam a variedade de atividades para avaliarem os alunos. Como afirma um dos entrevistados propicia a "interação síncrona no processo de ensino aprendizagem, a partir de chats e conversas pelo intercomunicador. Além disso, numa visão de coordenação, facilita o processo de gerenciamento e controle dos alunos dos cursos".

Pontos Negativos do AVEA: Os pontos fracos apontados foram muitos. O mais citado foi a complexidade do sistema: 3 (entrevistados): "é uma ferramenta bastante amarrada, que dificulta o acesso fácil às informações"; dois (2) mencionam a "lentidão do software quando há muitos alunos acessando", outros dois (2) nas atualizações (do sistema) há perda de algumas informações sobre os alunos; um (1) menciona o design da interface poluída, confusa; um (1) não permite acessar duas páginas simultaneamente; um (1) falhas no uso de som e imagem - "programa desconectava sem nenhum comando", 1 (um) rolagem do texto muito rápida gerando dificuldade para retornar a perguntas e comentários iniciais pois "o texto rola sozinho para o novo texto" e um (1) por afirmar que o sistema é terceirizado e para correção de erros, dependem do fornecedor - atraso nos processos internos e um (1) comenta sobre a "a falta de comunicação do ambiente virtual com o sistema de gestão do SENAI, gerando por exemplos problemas de acesso devido à duplicidade de usuários".

Ao serem questionados sobre o software utilizado, se ele proporciona o compartilhamento de informações necessário para a construção efetiva do conhecimento pretendido e, conforme a tabela 1 - Média de compartilhamento de informações - escala de 1 - discordo totalmente a 5 - concordo totalmente) apresentada a seguir, pode-se perceber que o AVEA utilizado pelos tutores oferece pouco compartilhamento das informações e alcançou médias abaixo de 2,5.

Tabela 1 - Média de compartilhamento de informações proporcionadas pelo software

\begin{tabular}{lc}
\hline \multicolumn{1}{c}{ Questão } & Média \\
O software consegue atender as minhas necessidades & 2,10 \\
As informações que me auxiliam no dia-a-dia do seu trabalho são armazenadas no & 2,21 \\
software e compartilhadas. & 2,0 \\
Com o software consigo muitas informações que facilitam o meu trabalho
\end{tabular}

Fonte: dados primários

Ao final da análise dos pontos negativos apontados, pôde-se perceber que os problemas do sistema no geral dificultam a troca das informações, em especial com a base de dados central do SENAI. Neste quesito, para haver conhecimento organizacional segundo Schendel (1996, p.83) é um fator "fundamental para a competitividade da empresa - é um recurso construído no decorrer da evolução da organização, sendo assim inimitável. É fundamental na construção de competências ou capacitações". Sugere-se melhorias neste quesito, como em outros apresentados abaixo.

Sugestões Propostas: Mais da metade dos respondentes - (6) seis - fez sugestões para modificações que minimizam ou eliminam pontos negativos por eles mesmos apontados . Mas, outros três (3) ainda mencionaram: (2 citaram) "mais videoconferência", 
"agilidade no suporte", "interatividade dos menus" e (1 citou) "customizar o sistema com o "cara do cliente". A seguir a Figura 2 apresenta um modelo de ferramenta interativa e síncrona: o chat - que foi mencionada pelos entrevistados.

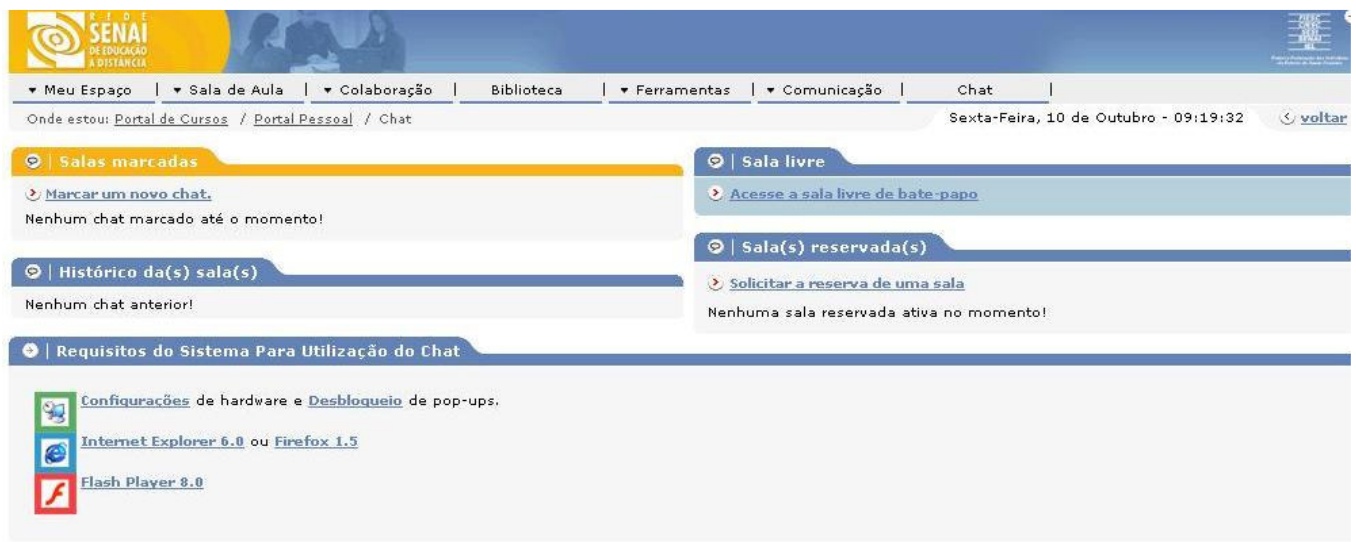

Figura 2 - Pagina Principal do chat no AVEA - Senai Virtual Fonte: (SENAI, 2008)

As principais dificuldades encontradas ao utlizarem o AVEA e que não são, segundo os entrevistados, reavaliadas e posteriormente incorporadas pela instituição em processo contínuo de retroalimentação (que poderiam ser feitos por meios de treinamentos constantes) são: "descobrir toda complexidade do sistema - "conhecer todas as ferramentas disponíveis"/ "adaptação ao sistema, tendo em vista o número maior de ferramentas disponíveis"/"informações novas passam desapercebidas"mencionado por quatro (4) entrevistados), um (1) uso das ferramentas síncronas ("som simultâneo, teclas a serem usadas para falar e liberar fala", um (1) "cadastro de alunos incompletos". Conforme depoimento de dois (2) entrevistados, para o ambiente novo comparando com o sistema já adotado, "teve que se ter um longo período de aprendizado e adaptação até conseguir que os usuários tivessem livre domínio das ferramentas do ambiente."

Ao serem questionados sobre as tendências de softwares para a Capacitação a Distância, houve uma unanimidade onde todos sugeriram ferramentas que proporcionem maior interatividade e facilidade (mais simples) de acesso, "que tenham mais interação no conteúdo, com os jogos, animações um conteúdo atrativo para os alunos, clientes" - "como o Second life" - por exemplo.

\section{Conclusões}

Ao apresentar as considerações finais, pode-se perceber que apesar do bom compartilhamento das informações sugere-se a maior adaptação do AVEA a partir das ações sugeridas por seus próprios membros - uma vez que no entendimento dos vários autores apresentados, é relevante compartilhar adequadamente as informações dentro de uma organização - possibilitando que suas partes consigam construir o conhecimento pretendido, ou seja, aprender com suas ações por meio da elaboração de um treinamento mais sistemático, conforme destacam Moore e Kearsley (2007). Neste ponto, ainda vale destacar o bom desempenho percebido decorrente do fato de a equipe entrevistada ser constantemente avaliada e receber feedback dos seus resultados.

Ao analisar os pontos positivos e negativos do AVEA percebe-se que o sistema não possibilita com facilidade os ajustes demandados, e que poderia ser incrementado 
pela redução da perda de informações (no momento de atualizar o sistema) e no incremento da interatividade, estratégias estas apontadas como tendências pela equipe.

Com o objetivo de promover um impacto significativo e mensurável nos resultados organizacionais, as variáveis que proporcionam a troca de informações e do conhecimento foram identificadas, mas precisam ser aperfeiçoadas, em especial no quesito de complexidade do sistema, na disponibilidade de ferramentas mais interativas e na análise da utilização das informações proporcionadas pelo ambiente (médias abaixo de 2,5). Ou seja, aplicar ao software sugestões mencionadas pelo grupo - que o utiliza diariamente - inserindo-os no processo de elaboração e/ou adaptação do ambiente (uma vez que além de usuários, eles também captam as dificuldades dos alunos).

No geral, a equipe de EAD caminha para atingir os quesitos (segundo fundamentação teórica apresentada) para ser uma Instituição que realiza a gestão e planejamento do AVEA e que necessita de ajustes inerentes ao processo de aperfeiçoamento que acontece em várias Instituições.

\section{REFERÊNCIAS BIBLIOGRÁFICAS}

BROWN, J. S.; DUIGUID, P. Equilibrismo: como capturar o conhecimento sem matálo. IN: REVIEW, H. B. Aprendizagem organizacional. Rio de Janeiro: Campus, 2001. CASTELLS, Manuel. A Sociedade em Rede. Rio de Janeiro, Paz e Terra, 1999.

CHOO, Chun Wei . A Organização do Conhecimento São Paulo : SENAC, 2003

DOUGIAMAS, M., Improving the effectiveness of tools for Internet based education. Teaching and Learning Forum 2000. Disponível em $<$ http://lsn.curtin.edu.au/tlf/tlf2000/dougiamas.html >Acesso em: 10 set. 2008

EVERS, Hans-Dieter. Towards a Malaysian Knowledge Society. Third International Malaysian Studies Conference (Msc3), Bangi,2001.

FLEURY, M. T.; OLIVEIRA JR, M. D. M. O. Gestão Estratégica do conhecimento: Integrando Aprendizagem, Conhecimento e Competências. São Paulo: Atlas, 2001, p. $50-85$.

MOORE, Michel G., KEARSLEY, Greg. Distance education: a systems view. Belmont (USA) : Wadsworth Publishing Company, 1973. $290 \mathrm{p}$.

SEED - SECRETARIA D EDUCACÃO A DISTÂNCIA MINISTÉRIO DA EDUCAÇÃO E CULTURA. Apresenta Textos sobre diretrizes em EAD. Disponível em:<http://portal.mec.gov.br/seed/index.php?option=com_content\&task=view\&id=105 54\&interna=6 $>$ e $<$ http://portal.mec.gov.br/seesp/index2.php?option=com_content\&do_ pdf $=1 \& \mathrm{id}=74>\quad$ - Acesso em : 20 out. 2008.

SCHENDEL, D. Knowledge and the firm. Strategic Management Journal. v. 17, n. Special Issue, 1996.

NAKAYAMA M.K., BINOTTO E., E PILLA, B.S. (org.). @-RH: Conceitos e Práticas de RH Eletrônico. Passo Fundo: Ed.Universidade de Passo Fundo, 2006.

OLIVEIRA, Eliane Regina da Costa. Um estudo comparativo entre o uso do computador e da videoconferência na educação à Distância. Biblioteca.Universia.. Florianópolis, EPS/UFSC 2000. (Tese doutorado).

RODRIGUES, Rosângela Schwarz. Modelo de Avaliação para cursos de Ensino a distância. Florianópolis, EPS/UFS, 1998.( (Tese doutorado).

SANTOS, Eduardo Toledo. Rodrigues, Marcos. Educação a Distância - conceitos, tecnologias, constatações, presunções e recomendações. São Paulo: EPUSP, 1999.32p

SENAI - Disponível em <www.sc.senai.br/senaivirtual >- Acesso em : 20 out. 2008.

SILVA, Reynaldo Pires. Uso das ferramentas síncronas e assíncronas nos cursos à distância. Disponível em: <http://www.ccuec.unicamp.br/ead/index_html?foco2= Publicacoes/78095/392385\&focomenu=Publicacoes $>$. Acesso em: 1 set. 2008.

SPODICK, E.F. The evolution of distance learning. Hong Kong: University of Science \& Tecnology Library. Revista do Centro de Computação e Comunicação - Chile Disponível em: http://sqzm14.ust.hk/distance. Acesso em: 23 out. 2008

TERRA, José Cláudio Cyrineu. GORDON, Cindy. Portais Corporativos: a revolução na gestão do conhecimento. São Paulo: Elsevier, 2002.

na gestão do conhecimento.São Paulo: Elsevier, 2002 